\title{
Automatic Lexical Acquisition Based on Statistical Distributions*
}

\author{
Suzanne Stevenson \\ Department of Computer Science \\ University of 'Joronto \\ 6 King's College Road \\ 'l'oronto, ON Canada M5S $3 \mathrm{H} 5$ \\ suzanne@cs , toronto. edu
}

\begin{abstract}
:
We automatically classily verbs into lexical semantic classes, based on distributions of indicators of verb alternations, extracted from a very largo annotated corpus. Wo address a problem which is particularly diflicult because the verb classes, although semantically different, show similar surface syntactic behavior. Tive grammatical features are sufficient to reduce error rate by more than 50\% over chance: we achieve almost $70 \%$ accuracy in a task whose baseline perlomance is $34 \%$, and whose expert-based upper bound we calculated at $86.5 \%$. We conclude that corpus-driven extraction of grammatical features is a promising methodology for fine-grained verb classification.
\end{abstract}

\section{Introduction}

Detailed information about verbs is critical to a broad range of NLP and IR tasks, yet its manual determination for large numbers of verlas is difficult, and resource intensive. Research on the automatic acquisition of verb-based knowledge las succeded in gleaning syntactic properties of verbs such as subcategorization frames from online resources (Brent, 1993; Briscoe and Carroll, 1997; Dorr, 1997; Manning, 1993). Recently, rescarchers have investigated statistical corpusbasod methods for lexical semantic classification from syntactic properties of verb usage (Aone and Mckee, 1996; Iapata and Brew, 1999; Schulte im Walde, 1998; Stevenson and Merlo, 1999; Stevenson et al., 1999; McCarthy, 2000).

Corpus-based approaches to lexical semantic classification in particular have drawn on Levin's hypothesis (Levin, 1993) that verbs can be classified according to the diathesis alternations (alternations in the syntactic expressions of arguments) in which they participate--for example, whether a

\footnotetext{
* This research was partly sponsored by US NSF grants \#9702331 and \#9818322, Swiss NST fellowship 821046569, Information Sciences Council of Rutgers University and IRCS, U. of Pemsylvania. This research was conducted while the first author was at Rutgers University.
}

\author{
Paola Merlo \\ J.A'T'T - Department of linguistics \\ University of Geneva. \\ 2 rue de Candolle \\ 1211 Genève 4 -.. Suisse \\ merlodlettres.unige.ch
}

verb occurs in the dative/prepositional phrase alternation in English. One diagnostic for diathesis alternations is the subcategorization alternatives of a verb. However, some classes exhibit the same subcategorization possibilitios but differ in their argument, structures, i.e. the content of the thematic roles assigned to the arguments of the verb. This type of situation constitutes a particularly diflicult case for corpus-based classification methods.

In this paper, we apply corpus-based lexical acquisition methoclology to distinguish classes of verbs which allow the same subcategorizations, but differ in thematic roles. We first assume that. one can antomatically restrict the choice of classes to those that participate in the relevant subcategorizations (cf. (Iapata and Brew, 1999)). Our proposal is then to use statistics over diathesis alternants as a way to further distinguish those verbs which allow the same subcategorizations, achicving finc-grained classification within that set. Our work focuses on determining the best semantic class for a verb lype- the set of usages of a verb across a document or corpus-rather than for a single verb token in a single local context. In this way, we can exploit the broad behavior of the verb across the corpus to determine its most likely classs overall.

We investigate the proposed approach in an indepth case study of the three major classes of optionally intransitive verbs in English: unergative, unaccusative, and object-drop. More specifically, according to Iovin's classification (levin, 1.993), the unergatives are manner of motion verbs, such as jump and march; the unaccusatives are verbs of change of state, such as open and explode; the object-drop verbs are unexpressed object alternation verlos, such as played and painted. These classes all support both transitive and intransitive subcategorizations, but are distinguished by the pattern of thematic role assignments to subject and object position. We automatically classify these verbs on the basis of statistical ap- 
proximations to syntactic indicators of the underlying argument structures, using numerical features collected from a large syntactically annotated (tagged or parsed) corpus. We apply machine learning techniques to dotermino whether the frequency distributions of the features, individually or in combination, support automatic classification of the verbs. To preview our results, we demonstrate that combining only five numerical indicators is sufficient to reduce the error rate in this classification task by more than $50 \%$ over chance. Specifically, we achieve almost $70 \%$ accuracy in a task whose baseline (chance) performance is $34 \%$, and whose expert-based upper bound is calculated at $86.5 \%$. We conclude that a distribution-based method for lexical scmantic verb classification is a promising avenue of research.

\section{The Argument Structures}

Our approach rests on the hypothesis that, even in cases where verb classes cannot be distinguished by subcategorizations, the frequency distributions of syntactic indicators can hold clues to the underlying thematic role differences. We start here then with a description of the subcategorizations and thematic role assignments for each of the three verb classes under investigation.

As optionally intransitive verbs, each of the three classes participates in the transitive/intransitive alternation:

Unergative

(1a) The horse raced past the barn.

(1b) The jockey raced the horse past the barn. Unaccusative

(2a) The butter melted in the pan.

(2b) The cook melted the butter in the pan.

Object-drop

(3a) The boy washed the hall.

(3b) The boy washed.

Unergatives are intransitive action verbs, as in (1), whose transitive form can be the causative counterpart of the intransitive form. In the causative use, the semantic argument that appears as the subject of the intransitive, as in (1a), surfaces as the object of the transitive, as in (1b) (Hale and Keyser, 1993). Unaccusatives are intransitive change of state verbs, as in (2a); the transitive counterpart for these verbs exhibits the causative alternation, as in (2b). Object-drop verbs, as in (3), have a non-causative transitive/intransitive alternation, in which the object is simply optional.

\begin{tabular}{|c|c|c|c|}
\hline Classes & $\begin{array}{l}\text { Sulbj of } \\
\text { Trans }\end{array}$ & $\begin{array}{l}\text { Obj of } \\
\text { Trans }\end{array}$ & $\begin{array}{l}\text { Subj of } \\
\text { Intrans }\end{array}$ \\
\hline Unergative & Causal Agent & Agent & Agent \\
\hline Unaccusative & Causal Agent & Theme & Theme \\
\hline Object-drop & Agent & Theme & Agent \\
\hline
\end{tabular}

Table 1: Summary of Thematic Alternations.

Each class is distinguished by the content of the thematic roles assigned by the verb. For objectdrop verbs, the subject is an Agent and the optional object is a Theme, yielding the thematic assignments (Agent, Theme) and (Agent) for the transitive and intransitive alternants sespectively. Unergatives and unaccusatives differ from objectdrop verbs in participating in the causative alternation, and also differ from cach other in their core thematic argument. In an intransitive uncrgative, the subject is an Agent, and in an intransitive unaccusative, the subject is a Theme. In the causative transitive form of each, this core semantic argument is expressed as the direct object, with the addition of a Causal $\Lambda$ gent (the causer of the action) as subject in both cases. 'The thematic roles assigned, and their mapping to syntactic position, are summarized in Table 1.

\section{The Features for Classification}

The key to any automatic classification task is to determine a set of useful features for discriminating the items to be classified. In what follows, we refer to the columns of Table $I$ to explain how we expect the thematic distinctions to yicld distributional features whose frequencies discriminate among the classes at hand.

Considering column one of Table 1, only unergative and unaccusative verbs assign a Causal Agent to the subject of the transitive. We hypothesize that the causative construction is linguistically more complex than the simple argument optionality of object-drop verbs (Stcvenson and Merlo, 1997). We expect then that objectdrop verbs will be more frequent in the transitive than the other two classes. Furthermore, the object of an unergative verb receives the Agent role (see the second column of Table 1), a linguistically marked transitive construction (Stcvenson and Merlo, 1997). We therefore expect unergatives to be quite rare in the transitive, leading to a three-way distinction in transitive usage among the three classes.

Second, due to the causative alternation of 
Table 2: The licatures and 'Their Wixpected Behavior

\begin{tabular}{|c|c|}
\hline Transitivity & $\begin{array}{l}\text { Unaccusatives and unergatives have a causative transitive, hence lower transitive use. Fur- } \\
\text { thermore, uncrgatives lave an agentive object, hence very low transitive use. }\end{array}$ \\
\hline Passive Voice & l'assive implics transitive use, licnce correlated with transitive feature. \\
\hline VIBN Tag & T'assive implics past participle use (VIBN), hence correlated with transitive (and passive). \\
\hline Cansativity & $\begin{array}{l}\text { Object-drop verlss do not have a causal agent, hence low "causative" use. Unergatives are } \\
\text { rare in the transitive, hence low causative use. }\end{array}$ \\
\hline Animacy & Unaccusatives have a theme subject in the intransitive, hence lower use of animate subjects. \\
\hline
\end{tabular}

unergatives and unaccusatives, the thematic role of the subject of the intransitive is identical to that of the object of the transitive, as shown in columns two and three of Table 1. Given the identity of thematic role mapped to subject and object positions, we expect to observe the same noun occurring at times as subject of the verb, and at other times as object of the verb. In contrast, for object-drop verbs, the thematic role of the subject of the intransitive is identical to that of the subject of the transitive, not, the object of the transitive. 'l'hus, we expect that it will be less common for the same noun to occur in subject and object position of the same object-drop verb. We hypothesize that this pattern of thematic role assignments will be reflected in differential amount of usage across the classes of the same nouns as subjects and objects for a given verb. Furthermore, since the cansative is a transitive use, and the transitive use of unergatives is expected to be rare, this overap of subjects and objects should primarily distinguish unaccusatives (predicted to have ligh overlap of subjects and objects) from the other two classes.

Finally, considering columns one and three of Table 1, we note that unergative and object-drop verbs assign an agentive role to their subject in both the transitive and intransitive, while unaccusatives assign an agentive role to their subject only in the transitive. Under the assumption that the intransitive use of unaccusatives is not rare, ${ }^{1}$ we then expect that unaccusatives will occur less often overall with an agentive subject than the other two verb classes. On the further assumption that Agents tend to be animate entitics more so than Themes, we expect that unaccusatives will occur less frequently with an animate subject compared to uncrgative and object-drop verbs. Note the importance of our use of frequency distributions: the claim is not that only Agents can

\footnotetext{
${ }^{1}$ This assumption is based on the linguistic complexity of the causative, and borne out in our corpus analysis.
}

be animate, but, rather that nouns that receive an Agent role will more often be animate than nouns that receive a 'Theme role.

The above interactions between thematic roles and the syntactic expressions of arguments thus lead to three features whose distributional properties appear promising for distinguishing the verb classes: transitivity, causativity, and animacy of subject. We also investigate two additional syntactic leatures, the passive voice and the past participle POS tag (VIBN). These features are related to the transitive/intransitive alternation, since a passive use implies a transitive use of the verb, and the use of passive in turn implies the use of the past participle. Our hypothesis is that these five fcatures will exhibit distributional differences in the observed usages of the verbs, which can be used for classification. The features and their expected relevance are summarized in Table 2.

\section{Data Collection and Analysis}

We chose a set of 20 verbs from cach of threc classes. The complete list of verbs is reported in Appendix A. Recall that our goal is to achieve a fine-grained classification of verbs that exhibit the same subcategorization frames; thus, the verbs were chosen because they do not generally show massive departures from the intended verb sense (and usage) in the corpus. ${ }^{2}$ In order to simplify the counting procedure, we included only the regular ("-ed") simple past/past participle form of the verb, assuming that this would approximate the distribution of the features across all forms of the verb. Finally, as far as we were able given the preceding constraints, we selected verbs that could occur in the transitive and in the passive.

We counted the occurrences of each verb token in a transitive or intransitive use (TRANS), in a

\footnotetext{
${ }^{2}$ Though note that there are only 19 unaccusatives bccause ripped was excluded from the analysis as it occurred mostly in a very different use (ripped off) in the corpus from the intended clange of state usage.
} 
passive or active use (PASS), in a past participle or simple past use (VBN), in a causative or noncausative use (CAUS), and with an animate subject or not (ANIM), as described below. The first three counts (TRANS, PASS, VBN) were performed on the LDC's 65-million word tagged ACL/DCI corpus (Brown, and Wall Street Journal 1987-1989). The last two counts (CAUS and ANIM) were performed on a 29-million word parsed corpus (Wall Street Journal 1988, provided by Michael Collins (Collins, 1997)). 'The features were counted as follows:

TRANS: The closest noun following a verb was considered a potential object. A verb immediately followed by a potential object was counted as transitive, otherwise as intransitive.

PASS: $\Lambda$ token tagged VBD (the tag for simple past) was counted as active. A token tagged VBN (the tag for past participle) was counted as active if the closest preceding auxiliary was have, and as passive if the closest preceding anxiliary was be.

VBN: The counts for VBN/VIBD were based on the POS label in the tagged corpus.

Each of the above counts was normalized over all occurrences of the "ed" form of the verb, yiclding a single relative frequency measure for each verb for that feature.

CAUS: For each verb token, the subject and object (if there was one) were extracted from the parsed corpus, and the proportion of overlap between subject and object nouns across all tokens of a verb was calculated.

ANIM: To approximate animacy without reference to a resource external to the corpus (such as WordNet), we count pronouns (other than it) in subject position (cf. (Aone and McKee, 1996)). The assumption is that the words $I$, we, you, she, he, and they most often refer to animate entities. We automatically extracted all subject/verb tuples, and computed the ratio of occurrences of pronoun subjects to all subjects for each verb.

The aggregate means by class resulting from the counts above are shown in Table 3. The distributions of each feature are indeed roughly as expected according to the description in Section 3. Unergatives show a very low relative frequency of the TRANS feature, followed by unaccusatives, then object-drop verbs. Unaccusative verbs show a high frequency of the caus feature and a low frequency of the ANIM feature compared to the other classes. Although expected to be a redundant indicator of transitivity, PASS and VBN do
Table 3: Aggregated Relative Frequency Data for the Five Features. $\mathrm{E}=$ unergatives, $\Lambda=$ unaccusatives, $\mathrm{O}=$ object-drops.

\begin{tabular}{|c|c|c|c|c|c|c|}
\hline \multirow[t]{2}{*}{ Class } & \multirow[t]{2}{*}{$\mathrm{N}$} & \multicolumn{5}{|c|}{ MEAN RELATIVE TREQUENCY } \\
\hline & & TRANS & PASS & VBN & CAUS & ANIM \\
\hline $\mathrm{E}$ & 20 & 0.23 & 0.07 & 0.21 & 0.00 & 0.25 \\
\hline$\Lambda$ & 19 & 0.40 & 0.33 & 0.65 & 0.12 & 0.07 \\
\hline $\mathrm{O}$ & 20 & 0.62 & 0.31 & 0.65 & 0.04 & 0.15 \\
\hline
\end{tabular}

not distinguish between unaccusative and objectdrop verbs, indicating that their distributions are sensitive to factors we have not yet investigated. ${ }^{3}$

\section{Experiments in Classification}

The frequency distributions of our features yield a vector for each verb that represents the rclative frequency values for the verb on each dimension: [verb, TRANS, PASS, vBN, CAUS, ANIM, class] Example: [opened, $.69, .09, .21, .16, .36$, unacc] We use the resulting 59 vectors to train an a.utomatic classifier to determine, given a verb that exhibits transitive/intransitive subcategorization frames, which of the three major lexical semantic classes of English optionally intransitive verbs it belongs to. Note that the baseline (chance) performance in this task is $33.9 \%$, since there are 59 vectors and 3 possible classes, with the most common class having 20 verbs.

We used the C5.0 machine learning system (http://www.rulequest.com), a nower version of C4.5 (Quinlan, 1992), which generates decision trees and corresponding rule sets from a training set of known classifications. We found little to no difference in performance between the trees and rule sets, and report only the rule set results. Wo report here on experiments using a single holdout training and testing methodology. In this approach, we hold out a single verb vector as the test case, and train the system on the remaining 58 cases. We then test the resulting classifier on the single hold-out case, recording the assigned class for that verb. This is then repeated for each of the 59 verbs. This technique has the benefit of yielding both an overall accuracy rate (when the results are averaged across all 59 trials), as well as providing the data necessary for determining accuracy for each verb class (because we have the classification of each verb when it is the test case). This allows us to evaluate the contribution

\footnotetext{
${ }^{3}$ These observations have been confirmed by t-tests between feature values for each pair of classes.
} 
Table 4: Percent Accuracy of Verb Classification Task Using lieatures in Combination. 'J'='RANS; $\mathrm{P}=\mathrm{P} \Lambda \mathrm{SS} ; \mathrm{V}=\mathrm{VBN} ; \quad \mathrm{C}=\mathrm{C} \Lambda \mathrm{US}$; $\Lambda \mathrm{n}=\Lambda \mathrm{NIM} . \quad \mathrm{E}=$ monergatives, $\Lambda=$ unaccusatives, $\mathrm{O}=$ object-drops

\begin{tabular}{|c|c|c|c|c|}
\hline & \multicolumn{4}{|c|}{ Percent Accuracy by Class } \\
\hline Features & All & $\bar{E}$ & $\Lambda$ & 0 \\
\hline 1.' I' V C An & 69.5 & 85.0 & 63.2 & 60.0 \\
\hline 2. $\mathrm{PV} C \overline{\Lambda n}_{\mathrm{n}}$ & $\overline{64 . \overline{1}}$ & 80.0 & $\overline{17 . \overline{4}}$ & 65.0 \\
\hline 3. T' V C $\mathrm{n}$ & 71.2 & 80.0 & 73.7 & 60.0 \\
\hline 4. T' $\mathrm{C} \mathrm{A}_{11}$ & 61.0 & 65.0 & 68.4 & 50.0 \\
\hline 5. 'I' P'V An & 62.7 & 70.0 & 63.2 & 55.0 \\
\hline 6. T P V C & 61.0 & 80.0 & 42.1 & 60.0 \\
\hline
\end{tabular}

of individual features with respect to their effect on the performance of individual classes.

We performed experiments on the full set of features, as woll as each subset of features with a single feature removed, as reported in Table 4. Consider the first column in the table. The first line shows that the overall accuracy for all five features is $69.5 \%$, a reduction in the error rate of more than $50 \%$ above the baseline. 'The removal of the pass feature appear's to improve performance (row 3 of lable 4). However, it should be noted that this increase in performance results from a single additional verb being classified correctly. The remaining rows show that no feature is superflous or hajmful as the removal of any feature has a 5 $8 \%$ negative effect on performance. Comparable accuracies have been demonstrated using a more thorough cross-validation methodology and using methods that are, in principle, better at taking advantage of correlated features (Stevenson and Merlo, 1999; Stevenson et al., 1999).

'T'he single hold-out protocol provides new data for andysing the performance on individual verbs and classes. The class-by-class accuracies are shown in the remaining columns of Table 4 . We can see clearly that, using all five features, the unergatives are classified with much greater accuracy $(85 \%)$ than the unaccusatives and objectdrop verbs $(63.2 \%$ and $60.0 \%$ respectively), as shown in the first, row. The remaining rows show that this pattern generally holds for the subsets of features as well, with the exception of line 4.

While future work on our verb classification task will need to focus on determining features that better discriminate unaccusative and objectdrop verbs, we can already exclude an explandation of the results based simply on the verbs' or the classes' frequency. Unergatives have the lowest average (log) frequency (1.3), but are the best classified, while unaccusatives and object-drops are comparable (average log frequency $=2$ ). If we group verbs by frequency, the proportion of errors to the total number of verbs remains fairly similar (freq 1: 7 crrors/23 verbs; freq. 2: 6 errors $/ 24$ verbs; freq. 3: 1 errors/10 verbs). The only verb of frequency 0 is correctly classified, while the only one with $\log$ frequency 4 is not. In sum, we do not find that more frequent classes or verbs are more accurately classified.

Importantly, the experiments also enable us to see whether the features indeed contribute to discriminating the classes in the manner predicted in Section 3. The single hold-out results allow us to do this, by comparing the individual class labels assigned using the full set of five features (TRANS, PASS, VBN, CAUS, ANIM) to the class labels assigned using each size four subset of features. This comparison indicates the changes in class labels that we can attribute to the added feature in going from a size four subset to the full set of features. (The individual class labels supporting our analysis below are available from the authors.) We concentrate on the three main features: CAUS, $\Lambda$ NM, TRANS. We find that the behaviour of these features generally does conform to our predictions. We expected that TrANs would help make a threeway distinction among the verb classes. While unergatives are already accurately classified without TR $\mathrm{N} s$, inspection of the change in class labels reveals that the addition of TRANS to the set improves performance on unaccusatives by helping to distinguish them from object-drops. IJowever, in this case, we also observe a loss in pre cision of unergatives, since some object-drops are now classified as unergatives. Morcover, we expected CAUS and $\Lambda$ NIM to bo particularly helpful in identifying unaccusatives, and this is also borne out in our analysis of individual labels. We note that the increased accuracy from CAUs is primarily due to better distinguishing unergatives from unaccusatives, and the increased accuracy from ANIM is primarily due to better distinguishing unaccusatives from object-drops. We conclude that the features we have devised are successful in clas sifing optionally transitive verbs because they capture predicted differences in underlying argument structure. ${ }^{4}$

\footnotetext{
${ }^{4}$ Matters are more complex with the other two features and we are still interpreting the results. Our prediction
} 
Table 5: Pair-wise Agreement (Calculated by the Kappa Statistics) of Three Experts (E1, E2, E3) Compared to a Gold Standard (Levin) and to the Classifier (Prog). Numbers in parentheses are percentage of verbs on which judges agree.

\begin{tabular}{|c|c|c|c|c|}
\hline & ProG & E1 & $\mathrm{I} 2$ & $\mathrm{~L} 3$ \\
\hline E 1 & $.36(59)$ & & & \\
\hline \pm 2 & $.50(68)$ & $59(75)$ & & \\
\hline $\mathrm{E} 3$ & $.49(66)$ & $.53(70)$ & $.66(77)$ & \\
\hline LEVIN & $.54(69.5)$ & $.56(71)$ & $.80(86.5)$ & $.74(83)$ \\
\hline
\end{tabular}

\section{Comparison to Experts}

In order to cvaluate the performance of the algorithm in practice, we need to compare it to the accuracy of classification performed by an expert, which gives a realistic upper bound for the task. In (Merlo and Stevenson, 2000) we report the results of an experiment that measures experts performance and agreement on a classification task very similar to the program we have described here. The results summarised in Table 5 illustrate the performance of the program. On the one hand, the algorithm does not perform at expert level, as indicated by the fact that, for all experts, the lowest agreement score is with the program. On the other hand, the accuracy achicved by the program of $69.5 \%$ is only $1.5 \%$ less than one of the human experts in comparison to the gold standasd. In fact, if we take the best performance achieved by an expert in this task$86.5 \%$ - as the maximum achievable accuracy in classification, our algorithm then reduces the error rate over chance by approximately $68 \%$, a very respectable result.

\section{Discussion}

The work here contributes both to general and technical issues in automatic lexical acquisition.

Firstly, our results confirm the primary role of argument structure in verb classification. Our experimental focus is particularly clear in this regard because we deal with verbs that are "mini-

was that VBN and PASS would behave similarly to TRANS. In fact, pASS is at best unhelpful in classification. VBN does appear to make the expected three-way distinction. The change in class labels shows that the improvement in performance with VBN results from better distinguishing unergatives from object-drops, and object-drops from unaccusatives. The latter is surprising, since analysis of the data found that the VBN feature values are statistically indistinct for the object-drop and unaccusative classes as a whole. mal pairs" with respect to argument structure. By classifying verbs that show the same subcategorizations into different classes, we are able to climinate one of the confounds in classification work created by the fact that subcategorization and argument structure are largely co-variant. We can infer that the accuracy in our classification is due to argument structure information, as subcategorization is the same for all verbs, confirming that the content of thematic roles is crucial for classification. Socondly, our results further support the assumption that thematic differences such as these are apparent not only in differences in subcategorization frames, but also in differences in their frequencies. We thus join the many recent results that all seem to converge in supporting the view that the relation between lexical syntax and semantics can be usefully exploited (Aone and McKee, 1996; Dorr, 1997; Dorr and Jones, 1996; Lapata and Brew, 1999; Schulte im Walde, 1998; Siegel, 1998), especially in a statistical framework. Finally, we observe that this information is detectable in a corpus and can be learned automatically. Thus we view corpora, especially if annotated with currently available tools, as useful repositories of implicit grammars.

Technically, our approach extends existing corpus-based learning techniques to a more complex learning problem, in several dimensions. Our statistical approach, which does not require explicit negative examples, extends approaches that cncode Icvin's alternations directly, as symbolic properties of a verb (Dorr et al., 1995; Dorr and Jones, 1996; Dorr, 1997). We also extend work using surface indicators to approximate underlying properties. (Oishi and Matsumoto, 1997) use case marking particles to approximate grammatical functions, such as subject and object. We improve on this approach by learning argument structure properties, which, unlike grammatical functions, are not marked morphologically. Others have tackled the problem of lexical semantic classification, as we have, but using only subcatcgorization frequencies as input data (Lapata and Brew, 1999; Schulte im Walde, 1998). By contrast, we explicitly address the definition of features that can tap directly into thematic role differences that are not reflected in subcategorization distinctions. Finally, when learning of thematic role assignment has been the explicit goal, the text has been semantically annotated (Webster and Marcus, 1989), or external semantic re- 
sources have been consulted (Aone and Mckee, 1996). We extend these results by showing that thematic information can be induced from corpus counts.

The experimental results show that our method is powerful, and suited to the classification of lexical items. However, we have not yet addressed the problem of verbs that can have multiple classifications. We think that many cases of ambiguous classification of verb types can be addressed with the notion of intersective sets introduced by (Dang et al., 1998). 'This is an important concept that proposes that "regular" ambiguity in classification -i.e., sets of verbs that have the same multi-way classifications according to (Jevin, 1993)-can be captured with a finergrained notion of lexical semantic classes. Fxtending our work to exploit this idea requires only to define the classes appropriately; the basic approach will remain the same. When we turn to consider ambiguity, we must also address the problem that individual instances of verbs may come from different classes. In future research wo plan to extend our method to the classification of ambiguous tokens, by experimenting with a function that combines several sources of information: a bias for the verb type (using the cross-corpus statistics we collect), as well as features of the usage of the instance being classified (cf. (I apata and Brew, 1999; Siegel, 1998)).

\section{References}

Chinatsu Aone and Douglas Mckee. 19396. Accuiring predicatc-argument, mapping information in multilingual texts. In Branimir Boguracv and Jamos P'ustejovsky, editors, Corpus Processing for Lexical Acquisition, pages 191-202. MIT Press.

Micluacl 3rent. 1993. From grammar to lexicon: Unsupervised lcarning of lexical syntax. Computational Linguistics, 19(2):243-262.

Ted Briscoe and John Carroll. 1997. Automatic extraction of subcategorization from corpora. In Procs of the lifth ANI P Conference, pages 356-363.

Michael John Collins. 1997. 'Three generative, Jexicalised models for statistical parsing. In Procs of $A C L ' 9 \%$, pages 16-23. Madrid, Spain.

Hoa 'Trang Dang, Karin Kipper, Martha l'almor, and Joseph Rosenzwcig. 1998. Investigating regular sense extensions based on intersective lovin classes. In Procs of OOLING-ACl, '98, pages 293-299, Montreal, Canlada.

Bonnic Dorr and Doug Jones. 1990. Role of word sense disambiguation in lexical acquisition: P'redicting scmantics from syntactic cues. In Proc. of COLING'96, pages 322-327, Copenhagen, Demmark.

Bomnic Jorr, Joe Garman, and Amy Weinberg. 1995. from syntactic encodings to thematic roles: Building lexical entries for interlingual MT'. Joarnal of Machine Translation, $9(3): 71 \cdots 1(0)$.

Bomic 1)orr. 1997. I arge-scale dictionary construction for forcign language tutoring and interlingual machine translation. Macluine Translation, 12:1-55.

Ken Hale and Jay Keyser. 1993. On argunent structure and the lexical representation of syntactic relations. In K. IJale and J. Kcyser, editors, The Vicw from Building 20, pages 53 1.10. MIT Press.

Maria Lapala and Chris J3rew. 1999. Using subcategorization to resolve verb class ambiguity. In Procs of Joint SIGDAT Conference on Entpirical Methods in Natural Language, College P'ark, MD.

Beth Levin. 1993. Inglish Verb Classes and Allernations. University of Chicago l'ress, Chicago, I]'.

Christopher I). Manning. 1993. Automatic acquisition of a large subcategorization dictionary from corpora. In Proes of ACL'93, pages 235-242. Ohio State University.

1)iana McCarthy. 2000. Using semantic preference to irlentify verly participation in role switching alternations. In Procs of $N A A C H$-2000, Seattle, Waslington.

Paola Merlo and Suzanne Stevenson. 2000.-Establishing the upper-bound and inter-judge agrement in a verb classification task. In P'rocs of LREC-2000, pages 16591664. Athens, Greece.

Akira Oishi and Yuji Matsumoto. 1997. Detecting the organization of scmantic subclasses of Japanese verbs. International Journal of Corpus linguistics, 2(1):65-89.

J. Ross Quinlan. 1992. O4.5: I'rograms for Machine Lcarning. Morgan Kaufmam, San Mateo, CA.

Sabine Schulte in Walde. 1998. Nutomatic semantic classification of verbs according to their alternation behaviour. AIMS Report 4(3), IMS, Universität Stuttgart.

thic V. Siegrel. 1998. Linguistic Indicators for language Understanding Ph.l). thesis, Dept. of Computer Scicuce, Columbia Universily.

Suzanne Stevenson and Paola Merlo. 1997. Jexical structure and processing complexity. Language and Cognitive Processes, $12(1-2): 349-399$.

Suzanne Stevenson and Paola Merlo. 1999. Verb classification using elistributions of granmatical features. In Procs of LACL'99. B Bergen, Norway.

Surame Stevenson, Paola Merlo, Natalia Kariacva, and Kamin Whitehouse. 1999. Supervised lcaming of lexical semantic verb classes using frequency distributions. In Procs of Siglex '99, College Park, Maryland.

Mort Wolsster and Mitch Marcus. 1989. Automatic acquisition of the lexical semantics of verlos from sentence frames. In Procs of ACL'89, pages 177-184, Vancouver, Canadia.

\section{Appendix A}

Unergatives: floated, galloped, glided, hiked, hopped, hurried, jogged, jumped, leaped, marched, paraded, raced, rushed, scoolcd, scurried, shipped, tiptocd, trotted, vaulted, wandered. Unaccusatives: boiled, changed, cleared, collapsed, coolcd, cracked, dissolved, divided, exploded, flooded, folded, fractured, hardened, melled, opened, simmered, solidified, slabilized, widened. Object-drops: borroved, called, carved, cleaned, danced, inherilod, kicked, knitled, organised, packed, painted, played, reaped, rented, slictched, studied, swallowed, lyped, washed, yelled. 